\title{
Adaptive Filtering of UAV Movement Parameters Based on AOA-Measurements of the Sensor Network in the Presence of Abnormal Measurements
}

\author{
Serhii Yakovych Zhuk ${ }^{1} \mathbb{D}$, Igor Olegovych Tovkach ${ }^{1, *}$ (D), Oleksandr Neuimin ${ }^{1} \mathbb{D}$, Volodymyr Vasyliev ${ }^{1}$
}

1. National Technical University of Ukraine Igor Sikorsky Kyiv - Polytechnic Institute Faculty of Radioengineering - Radio Engineering Devices and Systems - Kyiv/Kyiv - Ukraine

*Correspondence author: tovkach.igor@gmail.com

\begin{abstract}
The development and proliferation of small unmanned aerial vehicles (UAVs) have led to the need to create systems for tracking UAVs and monitoring their authorized activities. The presence of electromagnetic radiation makes it possible to use passive radio monitoring systems, based on wireless sensor networks, for tracking UAVs. Methods, based on angle-of-arrival (AOA) measurements, are widely used for determining the location of a radio source using wireless sensor networks. In practice, it becomes necessary to take into account the appearance of abnormal (rough) measurements, which lead to a sharp deterioration in the accuracy characteristics of Kalman filtration algorithms. In this work, to synthesize an optimal adaptive filtering algorithm, the Markov property of a mixed process was used, which includes a continuous-valued vector of UAV movement parameters and discrete parameters that characterize the type of measurements of the sensors of the sensor network. A quasi-optimal algorithm of adaptive filtering of UAV movement parameters when using AOA measurements of the sensor network was obtained using the Gaussian approximation method of the posterior probability density. Its analysis is carried out using a model example. The quasioptimal adaptive filtering algorithm allows to eliminate the uncontrolled increase of estimates errors of the UAV movement parameters and it does not require significant computational costs.
\end{abstract}

Keywords: UAV movement parameters; AOA measurements; Adaptive algorithm; Abnormal measurements; Wireless sensor networks.

\section{INTRODUCTION}

Currently, small unmanned aerial vehicles (UAVs) are widely used. The first reason for this is the miniaturization and cheapening of electronic components, such as processors, sensors, batteries, and wireless communication systems. The commercial UAV market opens wide access to this technology for private consumers, government and non-governmental organizations, and reduces the cost of their production while expanding capabilities and improving performance.

Despite the initial prerogative of using UAVs for solving military problems, technologies of small-sized UAVs are increasingly being used in various civilian spheres of activity: surveillance and reconnaissance, including in real time transportation and delivery

Received: Jun. 28, 2021 | Accepted: Oct 25, 2021

Peer Review History: Single Blind Peer Review.

Section Editor: Alison Moraes

This is an open access article distributed under the terms of the Creative Commons license. 
of goods and funds to a given area, relaying data between remote subscribers of communication networks; rescue operations; monitoring of emergency situations and their consequences; protection of objects and areas, etc.

However, this also led to the emergence of new threats to society (Lacher et al. 2019; U.S. Department of Homeland Security, 2020; Yaacoub et al. 2020): espionage, terrorism, transportation of prohibited goods, air traffic complications, property damage. Today, in the leading countries of the world, solving the problems of neutralizing threats from the use of small UAVs and creating appropriate protection systems have been brought to the level of national security. Therefore, tracking UAVs and monitoring of activities authorized for them are important tasks.

Due to the low visibility of UAVs, the tasks of their detection and tracking become much more complicated. An important feature of the UAV functioning is the presence of electromagnetic radiation in them. This allows passive radio monitoring systems based on wireless sensor networks (WSN) to be used to detect and tracking UAVs (Tang et al., 2008; Amiri et al. 2016; Chen and Wu 2018; Watanabe 2021).

A method, based on AOA measurements, is widely used in passive determination of a radio source coordinates (Hou et al. 2018; Tomic et al. 2018; Zhang et al. 2018). To determine the UAV coordinates in a rectangular coordinate system, it is sufficient to stablish its azimuths at two spaced points and the elevation angle at one of these points, or, conversely, the elevation angles at two receiving points and the azimuth at one of them. The WSN makes it possible to obtain a set of angular coordinates of the radio source at a fixed time instant, which makes it possible to solve the problem of joint processing of all available information and to improve the accuracy of determining the location of the radio source in space.

It is also known (Sirota and Kirsanov, 2006) that in real conditions, along with normal (usual) measurements, anomalous (rough) measurements may appear. Measurements with abnormal errors can be caused by the presence of signals with unknown parameters, an unknown number of radio sources, multipath signal propagation, the influence of interference. The appearance of abnormal measurements means a significant malfunction of WSN sensor (Kupriyanov and Sakharov, 2007; Tovkach et al. 2019; Zhuk et al. 2019; Tovkach and Zhuk 2021).

Discrete Kalman filtering is widely used to estimate the parameters of object movement. The main advantages of the Kalman filter is that it is recurrent and suitable for filtering non-stationary random signals.

However, the Kalman filter is operable only if there is complete a priori information about the process to be filtered. The presence of anomalous measurements, occurring at unknown times, leads to a significant excess of the variance of actual estimation errors over the variance of estimation errors calculated by the Kalman filter.

The optimal solution to the problem of adaptive filtering of UAV movement parameters, based on AOA measurements of the sensor network, resistant to the appearance of anomalous measurements, can be obtained using the Bayesian adaptive estimation method (Chang and Athans 1978). However, it cannot be implemented in practice due to the increase in the number of filter channels at each step. In the work (Bar-Sholom and Xivo Rong 1998), a quasi-optimal interactive multiple model (IMM) algorithm is obtained based on the Bayesian adaptive estimation method.

In this work, the mathematical apparatus of mixed Markov processes in discrete time (Zhuk, 2020), which allows obtaining optimal filters with a fixed number of channels and feedbacks between them, was used to solve the problem of adaptive filtering of UAV movement parameters based on AOA measurements of the sensor network. The quasi-optimal algorithm was obtained by a sequential method for processing incoming measurements, as well as Gaussian approximation of posterior probability density of estimated parameters.

\section{FORMULATION OF THE PROBLEM}

The UAV movement model in a rectangular coordinate system (CS) is described by the vector recurrent difference Eq. 1 (Tovkach et al. 2018):

$$
\grave{\mathbf{u}}(k)=\mathbf{F u}(k-1)+\mathbf{G} \quad(k)
$$

In which: 
$\mathbf{u}^{T}(k)=(x(k), \dot{x}(k), \ddot{x}(k), y(k), \dot{y}(k), \ddot{y}(k), \mathrm{z}(k), \dot{z}(k), \ddot{z}(k)):$ a state vector including coordinates of position, velocity and acceleration of the UAV in rectangular CS; $\mathbf{u}(k)$ : vector uncorrelated Gaussian excitation noise of a model with a unit correlation matrix; matrices $\mathbf{F}$ and have the form

$$
\begin{gathered}
\mathbf{F}=\left[\begin{array}{ccc}
\mathbf{F}^{b} & 0 & 0 \\
0 & \mathbf{F}^{b} & 0 \\
0 & 0 & \mathbf{F}^{b}
\end{array}\right] ; \mathbf{G}=\left[\begin{array}{ccc}
\mathbf{G}^{b} & 0 & 0 \\
0 & \mathbf{G}^{b} & 0 \\
0 & 0 & \mathbf{G}^{b}
\end{array}\right] ; \\
\mathbf{F}^{b}=\left[\begin{array}{ccc}
1 & T & \frac{T^{2}}{2} \\
0 & 1 & T \\
0 & 0 & 1
\end{array}\right] ; \mathbf{G}^{b}=\left[\begin{array}{c}
\frac{a \cdot T^{3}}{6} \\
\frac{a \cdot T^{2}}{2} \\
a \cdot T
\end{array}\right]
\end{gathered}
$$

In which:

$a$ : root mean square (RMS) of UAV acceleration change rate; $T$ : the rate of information receipt.

Eqs. 2, 3 and 4 are for the measurement of angular coordinates of UAV in the presence of abnormal measurements at the $k$-th step by pairs of sensors $S_{b i}, b=\overline{1, B}, i=\overline{0,1}$ :

$$
\begin{gathered}
\varphi_{b 0}^{M}(k)=\varphi_{b 0}(k)+a_{j_{b 0}}(k) \Delta \varphi_{b 0}(k) \\
\varphi_{b 1}^{M}(k)=\varphi_{b 1}(k)+a_{j_{b 1}}(k) \Delta \varphi_{b 1}(k) \\
\theta_{b 0}^{M}(k)=\theta_{b 0}(k)+a_{j_{b 0}}(k) \Delta \theta_{b 0}(k)
\end{gathered}
$$

In which:

$\varphi_{b 0}(k), \varphi_{b 1}(k), \theta_{b 0}(k)$ : true azimuths and elevation of UAV position in spherical CS (Fig. 1) of zero and first sensors of $b$-th pair; $b=\overline{1, B} ; \Delta \varphi_{b 0}(k), \Delta \varphi_{b 1}(k), \Delta \theta_{b 0}(k)$ : measurement errors of azimuths and variances $\sigma_{\varphi}^{2}$ and $\sigma_{\theta}^{2}$, respectively; $a_{j_{b i}}(k), j_{b i}=\overline{1,2}, b=\overline{1, B}, i=\overline{0,1}:$ switching variables, that take independent values with probabilities $q_{j_{b i}}, j_{b i}=\overline{1,2}, b=\overline{1, B}, i=\overline{0,1}$.

Switching variables characterize the type of sensor measurements. For the value of the indices $j_{b i}=1, b=\overline{1, B}, i=\overline{0,1}$ switching variables are $a_{j_{b i}}(k)=1$, which corresponds to normal measurement errors. For the value of the indices $j_{b i}=2$, $b=\overline{1, B}, i=\overline{0,1}$ switching variables are $a_{j_{b i}}(k)=\gamma$, in which $\gamma$ determines RMS value of errors $\sigma_{\varphi}, \sigma_{\theta}$ for abnormal measurements. In order to simplify calculations and without loss of generality, it is assumed that, if the reference sensor $S_{b 0}$ fails, abnormal measurements appear simultaneously when measuring the azimuth $\varphi_{b 0}(k)$ and elevation $\theta_{b 0}(k)$. 


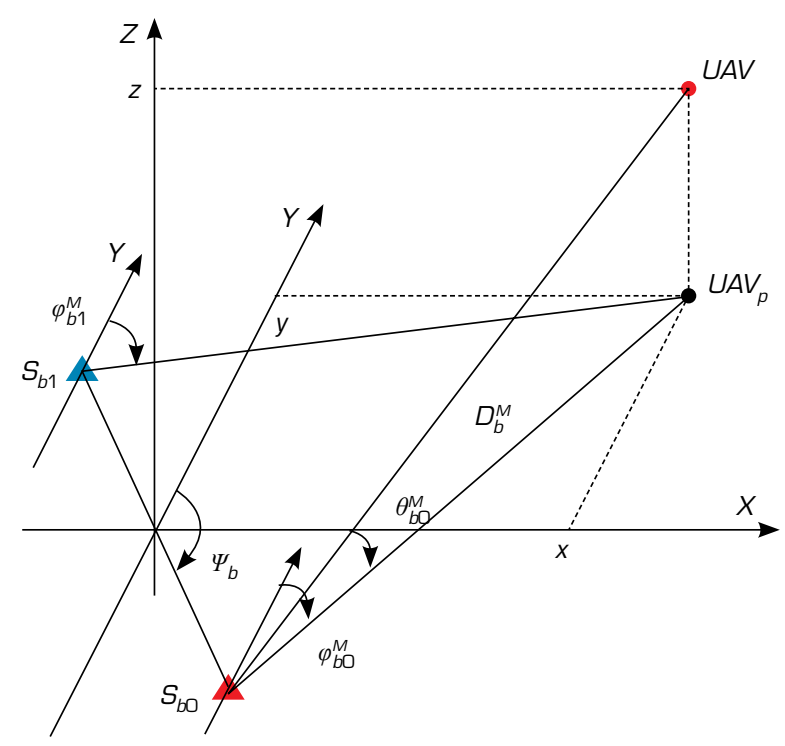

Figure 1. Relationship of unmanned aerial vehicle (UAV) coordinates in rectangular coordinate system and angular measurements by sensors pair $S_{b i}, i=0,1$.

Based on measurements of sensor pairs $S_{b i}, b=1, \bar{B}, i=\overline{0,1}(2,3$ and 4$)$, UAV coordinates in rectangular CS are determined by Eqs. 5, 6 and 7 (Tovkach and Zhuk, 2020):

$$
\begin{aligned}
& x_{b}^{M}(k)=D_{b}^{M}(k) \sin \varphi_{b 0}^{M}(k) \\
& y_{b}^{M}(k)=D_{b}^{M}(k) \cos \varphi_{b 0}^{M}(k) \\
& z_{b}^{M}(k)=D_{b}^{M}(k) \tan \theta_{b 0}^{M}(k)
\end{aligned}
$$

In which:

$D_{b}^{M}(k)$ : the distance projection from zero sensor $b$-th pair of $S_{b 0}$ to the target on the XY plane, which is calculated by Eq. 8 (Tovkach et al. 2020):

$$
D_{b}^{M}(k)=\frac{d_{b} \sin \left(180-\left(\psi_{b}-\varphi_{b 1}^{M}(k)\right)\right)}{\sin \left(\left(\psi_{b}-\varphi_{b 1}^{M}(k)\right)-\left(\psi_{b}-\varphi_{b 0}^{M}(k)\right)\right)}
$$

$d_{b}$ : the distance between the sensors of the $b$-th pair, which is determined by Eq. 9.

$$
d_{b}=\sqrt{\left(x_{b 0}-x_{b 1}\right)^{2}+\left(y_{b 0}-y_{b 1}\right)^{2}}
$$

Coordinate values $x_{b}^{M}(k), y_{b}^{M}(k), z_{b}^{M}(k)$ and projections $D_{b}^{M}(k)$ depend on the values that the corresponding switching variables $a_{j_{b 0}}(k), a_{j_{b 1}}(k)$, take.

Equations 5, 6 and 7 contain trigonometric functions and are nonlinear.

The linearized observation equation in the local rectangular CS is Eq. 10.

$$
\mathbf{u}_{b}^{M}(k)=\mathbf{H}_{1} \mathbf{u}(k)+\Delta \mathbf{u}_{b}^{M}\left(a_{j_{b 0}}(k), a_{j_{b 1}}(k)\right)
$$


In which:

$\mathbf{u}_{b}^{M}(k)=\left(x_{b}^{M}(k), y_{b}^{M}(k), z_{b}^{M}(k)\right)^{T}$ : the vector of target coordinates measurement in rectangular CS; $\Delta \mathbf{u}_{b}^{M}\left(a_{j_{b 0}}(k), a_{j_{b 1}}(k)\right)=$ $=\left(\Delta x_{b}^{M}\left(a_{j_{b 0}}(k), a_{j_{b 1}}(k)\right), \Delta y_{b}^{M}\left(a_{j_{b 0}}(k), a_{j_{b 1}}(k)\right), \Delta z_{b}^{M}\left(a_{j_{b 0}}(k), a_{j_{b 1}}(k)\right)\right)^{T}$ : the vector of measurement errors of target coordinates in a rectangular CS with a correlation matrix $\mathbf{R}_{j_{b 0} j_{b 1}}(k)$.

$$
\mathbf{H}_{1}=\left[\begin{array}{lllllllll}
1 & 0 & 0 & 0 & 0 & 0 & 0 & 0 & 0 \\
0 & 0 & 0 & 1 & 0 & 0 & 0 & 0 & 0 \\
0 & 0 & 0 & 0 & 0 & 0 & 1 & 0 & 0
\end{array}\right] .
$$

Elements of the correlation matrix $\mathbf{R}_{j_{b 0} j_{b 1}}(k)$ are calculated by Eqs. 11-16.

$$
\begin{gathered}
R_{11}(k)=\sigma_{\varphi}^{2}\left(a_{j_{b 0}}^{2}(k) \alpha_{1}^{2}(k)+a_{j_{b 1}}^{2}(k) \alpha_{2}^{2}(k)\right) \\
R_{22}(k)=\sigma_{\varphi}^{2}\left(a_{j_{b 0}}^{2}(k) \beta_{1}^{2}+a_{j_{b 1}}^{2}(k) \beta_{2}^{2}\right) \\
R_{33}(k)=a_{j_{b 0}}^{2}(k)\left(\sigma_{\varphi}^{2}\left(\gamma_{1}^{2}+\gamma_{2}^{2}\right)+\sigma_{\theta}^{2} \gamma_{3}^{2}\right) \\
R_{12}(k)=R_{21}(k)=\sigma_{\varphi}^{2}\left(a_{j_{b 0}}^{2}(k) \alpha_{1} \beta_{1}+a_{j_{b 1}}^{2}(k) \alpha_{2} \beta_{2}\right) \\
R_{13}(k)=R_{31}(k)=\sigma_{\varphi}^{2}\left(a_{j_{b 0}}^{2}(k) \alpha_{1} \gamma_{1}+a_{j_{b 1}}^{2}(k) \alpha_{1} \gamma_{2}\right) \\
R_{23}(k)=R_{32}(k)=\sigma_{\varphi}^{2}\left(a_{j_{b 0}}^{2}(k) \beta_{1} \gamma_{1}+a_{j_{b 1}}^{2}(k) \beta_{1} \gamma_{2}\right)
\end{gathered}
$$

In which:

$\alpha_{1}(k), \alpha_{2}(k), \beta_{1}(k), \beta_{2}(k)$, coefficients determined by Eqs. 17-25:

$$
\begin{gathered}
\alpha_{1}(k)=c_{1}(k) \sin \varphi_{b 0}+D_{b}(k) \cos \varphi_{b 0} \\
\alpha_{2}=c_{2} \sin \varphi_{b 0} \\
\beta_{1}=c_{1} \cos \varphi_{b 0}+D_{b} \sin \varphi_{b 0} \\
\beta_{2}=c_{2} \cos \varphi_{b 0} \\
\gamma_{1}=c_{1} \tan \theta_{b 0} \\
\gamma_{2}=c_{2} \tan \theta_{b 0} \\
\gamma_{3}=D_{b} \frac{1}{\cos ^{2} \theta_{b 0}}
\end{gathered}
$$




$$
\begin{aligned}
& c_{1}=-\frac{b_{b} \sin \left(180-\left(\alpha_{b}-\varphi_{b 1}\right)\right) \cos \left(\left(\alpha_{b}-\varphi_{b 1}\right)-\left(\alpha_{b}-\varphi_{b 0}\right)\right)}{\sin \left(\left(\alpha_{b}-\varphi_{b 1}\right)-\left(\alpha_{b}-\varphi_{b 0}\right)\right)^{2}} \\
& c_{2}=\frac{b_{b}}{\sin ^{2}\left(\left(\alpha_{b}-\varphi_{b 1}\right)-\left(\alpha_{b}-\varphi_{b 0}\right)\right)}\left(\cos \left(180-\left(\alpha_{b}-\varphi_{b 1}\right)\right) \times\right. \\
& \times \sin \left(\left(\alpha_{b}-\varphi_{b 1}\right)-\left(\alpha_{b}-\varphi_{b 0}\right)\right)+ \\
& \left.+\sin \left(180-\left(\alpha_{b}-\varphi_{b 1}\right)\right) \cos \left(\left(\alpha_{b}-\varphi_{b 1}\right)-\left(\alpha_{b}-\varphi_{b 0}\right)\right)\right) .
\end{aligned}
$$

In order to reduce the size in the designations of the elements of the correlation matrix, their dependence on the values of the switching variables $a_{j_{b 0}}(k), a_{j_{b 1}}(k)$, is not indicated.

The UAV movement model (1) and measurement Eqs. (10) describe a priori data, necessary for the synthesis of optimal and quasi-optimal algorithms of adaptive filtering of the UAV movement parameters, based on AOA measurements of the sensor network in the presence of anomalous measurements. In this case, together with the estimation of UAV movement parameters, the problem of measurement type recognition should be solved. The results of measurement type recognition are used in the formation of estimates of the UAV movement parameter vector.

\section{DEVELOPMENT OF OPTIMAL AND QUASI-OPTIMAL ADAPTIVE FILTERING ALGORITHMS}

The minimum criterion of the RMS of error is widely used in solving the filtering problem (Sage and Mels, 1971). The optimal estimate of the state vector in accordance with this criterion is the conditional expected values of its a posteriori probability density (p.d.f.). The correlation matrix of a posteriori p.d.f. of the state vector is the correlation matrix of the estimation errors.

Enter the vector $\mathbf{u}_{b}^{M}(k)=\left(x_{b}^{M}(k), y_{b}^{M}(k), z_{b}^{M}(k)\right)^{T}$, including UAV coordinates in a rectangular CS, calculated by measurements of a sensors pair $S_{b i}, i=\overline{0,1}$. UAV coordinates, calculated from all sensor pairs $S_{b i}, b=1, B, i=\overline{0,1}$, denote as a vector $\mathbf{u}^{M}(k)=\left(\mathbf{u}_{1}^{M}(k), \ldots, \mathbf{u}_{B}^{M}(k)\right)^{T}$.

Combine Eq. 10 into Eq. 26.

$$
\mathbf{u}^{M}(k)=\mathbf{H} \mathbf{u}^{M}(k)+\Delta \mathbf{u}^{M}(\boldsymbol{\Gamma}(k))
$$

In which:

$\Delta \mathbf{u}^{M}(\boldsymbol{\Gamma}(k))=\left(\Delta \mathbf{u}_{1}^{M}\left(a_{j_{10}}(k), a_{j_{11}}(k)\right), \ldots, \Delta \mathbf{u}_{B}^{M}\left(a_{j_{B 0}}(k), a_{j_{B 1}}(k)\right)\right)^{T}:$ combined vector of target coordinates measurement in rectangular CS with correlation matrix; $\Gamma(k)=\left(a_{j_{10}}(k), a_{j_{11}}(k), . ., a_{j_{B 0}}(k), a_{j_{B 1}}(k)\right)$ : combined vector of switching variables.

$$
\mathbf{R}_{\Gamma}(k)=\left[\begin{array}{ccc}
\mathbf{R}_{j_{10} j_{11}}(k) & \ldots & 0 \\
\ldots & \mathbf{R}_{j_{b 0} j_{b 1}}(k) & \ldots \\
0 & \ldots & \mathbf{R}_{j_{B 0} j_{B 1}}(k)
\end{array}\right] ; \mathbf{H}=\left[\begin{array}{ccc}
\mathbf{H}_{1} & \ldots & 0 \\
\ldots & \mathbf{H}_{1} & \ldots \\
0 & \ldots & \mathbf{H}_{1}
\end{array}\right]
$$


Introduce an extended mixed process $(\mathbf{u}(k), \boldsymbol{\Gamma}(k))$. In Zhuk (2020), it is shown that the mixed process $(\mathbf{u}(k), \boldsymbol{\Gamma}(k))$ possesses the Markov property. Taking into account that the discrete components $\boldsymbol{\Gamma}(k)$ are independent, a posteriori p.d.f. of the mixed process $(\mathbf{u}(k), \boldsymbol{\Gamma}(k))$ is recurrently calculated by the Eqs. 27,28 and 29.

$$
\begin{aligned}
& P\left(\mathbf{u}(k), \boldsymbol{\Gamma}(k) \mid \mathbf{U}^{M}(k-1)\right)=\prod_{b=1}^{B} q_{j_{b 0}} q_{j_{b 1}} \times \\
& \times \int_{-\infty}^{\infty} \Pi(\mathbf{u}(k) \mid \mathbf{u}(k-1)) P\left(\mathbf{u}(k-1) \mid \mathbf{U}^{M}(k-1)\right) d \mathbf{u}(k-1) ; \\
& P\left(\mathbf{u}(k), \boldsymbol{\Gamma}(k) \mid \mathbf{U}^{M}(k)\right)=P\left(\mathbf{u}^{M}(k) \mid \mathbf{u}(k), \boldsymbol{\Gamma}(k)\right) \times \\
& \times P\left(\mathbf{u}(k), \boldsymbol{\Gamma}(k) \mid \mathbf{U}^{M}(k-1)\right) / P\left(\mathbf{u}^{M}(k) \mid \mathbf{U}^{M}(k-1)\right) ; \\
& P\left(\mathbf{u}(k) \mid \mathbf{U}^{M}(k)\right)=\sum_{j_{10}=1}^{2} \sum_{j_{11}=1}^{2} \ldots \sum_{j_{B 0}=1}^{2} \sum_{j_{B 1}=1}^{2} P\left(\mathbf{u}(k), \boldsymbol{\Gamma}(k) \mid \mathbf{U}^{M}(k)\right),
\end{aligned}
$$

In which:

$P\left(\mathbf{u}(k), \boldsymbol{\Gamma}(k) \mid \mathbf{U}^{M}(k-1)\right)$ : extrapolated p.d.f. of the mixed process $(\mathbf{u}(k), \boldsymbol{\Gamma}(k)) ; \mathbf{U}^{M}(k-1)=\left(\mathbf{u}^{M}(1), \ldots, \mathbf{u}^{M}(k-1)\right)$ : received up to $k-1$ step inclusive sequence of measurements; $\Pi(\mathbf{u}(k) \mid \mathbf{u}(k-1))$ : conditional p.d.f., which is found on the basis of Eq. 1; $P\left(\mathbf{u}(k), \boldsymbol{\Gamma}(k) \mid \mathbf{U}^{M}(k)\right)$ : a posteriori p.d.f. of mixed process $(\mathbf{u}(k), \boldsymbol{\Gamma}(k))$; $P\left(\mathbf{u}^{M}(k) \mid \mathbf{u}(k), \boldsymbol{\Gamma}(k)\right)=\prod_{b=1}^{B} P\left(\mathbf{u}_{b}^{M}(k) \mid \mathbf{u}(k), a_{j_{0}}(k), a_{j_{b 1}}(k)\right)$ : likelihood function of the observation vector $\mathbf{u}^{M}(k)$, which is based on the Eqs. 10 and 26; $P\left(\mathbf{u}^{M}(k) \mid \mathbf{U}^{M}(k-1)\right)$ : conditional p.d.f., which plays the role of a normalizing factor, which is calculated by Eq. 30 .

$$
\begin{gathered}
P\left(\mathbf{u}^{M}(k) \mid \mathbf{U}^{M}(k-1)\right)=\sum_{j_{10}=1}^{2} \sum_{j_{11}=1}^{2} \ldots \sum_{j_{B 0}=1}^{2} \sum_{j_{B 1}=1}^{2} \int_{-\infty}^{\infty} P\left(\mathbf{u}^{M}(k) \mid \mathbf{u}(k), \boldsymbol{\Gamma}(k)\right) \times \\
\times P\left(\mathbf{u}(k), \boldsymbol{\Gamma}(k) \mid \mathbf{U}^{M}(k-1)\right) d u(k) ;
\end{gathered}
$$

$P\left(\mathbf{u}(k) \mid \mathbf{U}^{M}(k)\right)$ is a posteriori p.d.f. of the vector, determined at the $k$-th step. The initial condition for algorithm (27) ... (29) is a priori p.d.f. $P(\mathbf{u}(0))$.

Eqs. 27, 28 and 29 describe the algorithm for joint optimal nonlinear filtering of the state vector $\mathbf{u}(k)$ and discrete components $\boldsymbol{\Gamma}(k)$. The algorithm is recurrent. Using a posteriori p.d.f. $P\left(\mathbf{u}(k-1) \mid \mathbf{U}^{M}(k-1)\right)$, obtained at the step $k$-1 using the Eq. 27, the problem of joint prediction of the values of discrete components $\boldsymbol{\Gamma}(k)$ and the vector $\mathbf{u}(k)$ is solved at the $k$-th step, and the extrapolated p.d.f. $P\left(\mathbf{u}(k), \boldsymbol{\Gamma}(k) \mid \mathbf{U}^{M}(k-1)\right)$ is calculated. Based on the obtained measurements $\mathbf{u}^{M}(k)$, using Eq. 28, a posteriori p.d.f. $P\left(\mathbf{u}(k), \boldsymbol{\Gamma}(k) \mid \mathbf{U}^{M}(k)\right)$ of the mixed process $(\mathbf{u}(k), \boldsymbol{\Gamma}(k))$ is calculated. Using Eq. 29, a posteriori p.d.f. $P\left(\mathbf{u}(k) \mid \mathbf{U}^{M}(k)\right)$ of the vector $\mathbf{u}(k)$ is calculated. The procedure is then repeated.

However, the implementation of an optimal real-time mixed process filtering algorithm encounters significant difficulties associated with the need to integrate multidimensional p.d.f. and a large number of tested hypotheses regarding the values of switching variables.

When synthesizing a quasi-optimal algorithm, we used the Gaussian approximation method of a posteriori p.d.f. vector $\mathbf{u}(k)$. Let the a posteriori p.d.f. $P\left(\mathbf{u}(k-1) \mid \mathbf{U}^{M}(k-1)\right)$, obtained at the $k$-1-th step, be Gaussian with expected values $\hat{\mathbf{u}}(k-1)$ 
and correlation matrix $\hat{\mathbf{P}}(k-1)$. The extrapolated p.d.f. $P\left(\mathbf{u}(k) \mid \mathbf{U}^{M}(k-1)\right)$ of vector, based on Eq. 1, was determined by Eq. 31 .

$$
P\left(\mathbf{u}(k) \mid \mathbf{U}^{M}(k-1)\right)=\int_{-\infty}^{\infty} \Pi(\mathbf{u}(k) \mid \mathbf{u}(k-1)) P\left(\mathbf{u}(k-1) \mid \mathbf{U}^{M}(k-1)\right) d \mathbf{u}(k-1)
$$

Since p.d.f. $\Pi(\mathbf{u}(k) \mid \mathbf{u}(k-1))$ and $P\left(\mathbf{u}(k-1) \mid \mathbf{U}^{M}(k-1)\right)$ are Gaussian, extrapolated p.d.f. $P\left(\mathbf{u}(k) \mid \mathbf{U}^{M}(k-1)\right)$ is also Gaussian, and its mathematical expectation $\mathbf{u}^{*}(k)$ and correlation matrix $\mathbf{P}^{*}(k)$ are calculated by Eqs. 32 and 33.

$$
\begin{gathered}
\mathbf{u}(k)=\mathbf{F} \hat{\mathbf{u}}(k-1) \\
\mathbf{P}^{*}(k)=\mathbf{F} \hat{\mathbf{P}}(k-1) \mathbf{F}^{T}+\mathbf{G}_{j_{0}} \mathbf{G}_{j_{0}}^{T}
\end{gathered}
$$

We used a sequential method (Tovkach and Zhuk 2019) for processing of incoming data. In this case, when the next measurement $\mathbf{u}_{b}^{M}(k)$ arrives, a Gaussian approximation of a posteriori p.d.f. $P\left(\mathbf{u}(k) \mid \mathbf{u}_{1}^{M}(k), \ldots, \mathbf{u}_{b}^{M}(k), \mathbf{U}^{M}(k-1)\right), b=\overline{1, B}$ is performed. In this case, the conditional a posteriori p.d.f. $P\left(\mathbf{u}(k) \mid a_{j b 0}(k), a_{j b 1}(k), \mathbf{u}_{1}^{M}(k), \ldots, \mathbf{u}_{b}^{M}(k), \mathbf{U}^{M}(k-1)\right)$, $j_{b 0}, j_{b 1}=1,2$, refined by measurements $\mathbf{u}_{b}^{M}(k)$, are Gaussian. Their expected values $\hat{\mathbf{u}}_{j_{b_{0}} j_{b_{1}}}(k)$ and correlation matrices $\hat{\mathbf{P}}_{b_{b_{0}} j_{b_{1}}}(k)$ are determined by Eqs. 34, 35 and 36 .

$$
\begin{gathered}
\mathbf{K}_{j_{b_{0}} j_{b_{1}}}(k)=\hat{\mathbf{P}}_{b-1}(k) \mathbf{H}_{1}^{T}\left(\mathbf{H}_{1} \hat{\mathbf{P}}_{b-1}(k) \mathbf{H}_{1}^{T}+\mathbf{R}_{j_{b_{0}} j_{b_{1}}}(k)\right)^{-1} ; \\
\hat{\mathbf{u}}_{j_{b_{0}} j_{b_{1}}}(k)=\hat{\mathbf{u}}_{b-1}(k)+\mathbf{K}_{j_{b_{0}} j_{b_{1}}}(k)\left(\mathbf{u}_{b}^{M}(k)-\mathbf{H}_{1} \hat{\mathbf{u}}_{b-1}(k)\right) \\
\hat{\mathbf{P}}_{j_{b_{0}} j_{b_{1}}}(k)=\hat{\mathbf{P}}_{b-1}(k)-\mathbf{K}_{j_{b_{0}} j_{b_{1}}}(k) \mathbf{H}_{1} \hat{\mathbf{P}}_{b-1}(k)
\end{gathered}
$$

In which:

$\mathbf{K}_{j_{b_{0}} j_{b_{1}}}(k)$ : the gains of the adaptive filter channels, matched with the corresponding types of measurement errors .

The joint posterior probability $P\left(a_{j b 0}(k), a_{j b 1}(k) \mid \mathbf{u}_{1}^{M}(k), \ldots, \mathbf{u}_{b}^{M}(k), \mathbf{U}^{M}(k-1)\right)$ of the switching variables values $a_{j_{b 0}}(k), a_{j_{b 1}}(k)$, refined by measurements $\mathbf{u}_{b}^{M}(k), b=\overline{1, B}$, was determined by Eq. 37 .

$$
\begin{aligned}
& P\left(a_{j_{b 0}}(k), a_{j_{b 1}}(k) \mid \mathbf{u}_{1}^{M}(k), \ldots, \mathbf{u}_{b}^{M}(k), \mathbf{U}^{M}(k-1)\right)= \\
& =P\left(\mathbf{u}_{b}^{M}(k) \mid a_{j_{b 0}}(k) a_{j_{b 1}}(k), \mathbf{u}_{1}^{M}(k), \ldots, \mathbf{u}_{b-1}^{M}(k), \mathbf{U}^{M}(k-1)\right) \times \\
& \times q_{j_{b 0}} q_{j_{b 1}} / P\left(\mathbf{u}_{b}^{M}(k) \mid \mathbf{u}_{1}^{M}(k), \ldots, \mathbf{u}_{b-1}^{M}(k), \mathbf{U}^{M}(k-1)\right) .
\end{aligned}
$$

The likelihood function of the values of the switching variables $a_{j_{b 0}}(k), a_{j_{b 1}}(k)$ is Gaussian. 


$$
\begin{gathered}
\left.\mathrm{P}\left(\mathbf{u}_{b}^{M}(k) \mid a_{j_{b 0}}(k), a_{j_{b 1}}(k), \mathbf{u}_{1}^{M}(k), \ldots, \mathbf{u}_{b-1}^{M}(k), \mathbf{U}^{M}(k-1)\right)\right)= \\
=N\left(\mathbf{H}_{1} \hat{\mathbf{u}}_{b-1}, \mathrm{D}_{j_{b_{0}} j_{b_{1}}}(k)\right),
\end{gathered}
$$

In which:

$\mathrm{D}_{j_{b_{0}} j_{b_{1}}}(k)$ : correlation matrix of the residual, determined by Eq. 39.

$$
\mathrm{D}_{j_{b_{0}} j_{b_{1}}}(k)=\mathbf{H}_{1} \hat{\mathbf{P}}_{b-1}(k) \mathbf{H}_{1}^{T}+\mathbf{R}_{j_{b_{0}} j_{b_{1}}}(k) .
$$

A posteriorip.d.f. $P\left(\mathbf{u}(k) \mid \mathbf{u}_{1}^{M}(k), \ldots, \mathbf{u}_{b}^{M}(k), \mathbf{U}^{M}(k-1)\right)$ is the weighted with a posterioriprobabilities $P\left(a_{j_{b 0}}(k), a_{j_{b 1}}(k) \mid \mathbf{u}_{1}^{M}(k), \ldots\right.$, $\left.\mathbf{u}_{b}^{M}(k), \mathbf{U}^{M}(k-1)\right)$ sum of the conditional a posteriori Gaussian p.d.f. $P\left(\mathbf{u}(k) \mid a_{j_{b 0}}(k), a_{j_{b 1}}(k), \mathbf{u}_{1}^{M}(k), \ldots, \mathbf{u}_{b}^{M}(k), \mathbf{U}^{M}(k-1)\right)$. Its expected values $\hat{\mathbf{u}}_{b}(k)$ and correlation matrix were determined by Eqs. 40 and 41.

$$
\begin{gathered}
\hat{\mathbf{u}}_{b}(k)=\sum_{j_{b 0}=1}^{2} \sum_{j_{b 1}=1}^{2} \hat{\mathbf{u}}_{j_{b_{0}} j_{b_{1}}}(k) P\left(a_{j_{b 0}}(k), a_{j_{b 1}}(k) \mid \mathbf{u}_{1}^{M}(k), \ldots, \mathbf{u}_{b}^{M}(k), \mathbf{U}^{M}(k-1)\right) \\
\hat{\mathbf{P}}_{b}(k)=\sum_{j_{b 0}=1}^{2} \sum_{j_{b 1}=1}^{2} \hat{\mathbf{P}}_{j_{b_{0}} j_{b_{1}}}(k) P\left(a_{j_{b 0}}(k), a_{j_{b 1}}(k) \mid \mathbf{u}_{1}^{M}(k), \ldots, \mathbf{u}_{b}^{M}(k), \mathbf{U}^{M}(k-1)\right), b=\overline{1, B}
\end{gathered}
$$

The initial conditions for Eqs. 34-41 at $b=0$ have the form $\hat{\mathbf{u}}_{0}(k)=\mathbf{u}^{*}(k), \hat{\mathbf{P}}_{0}(k)=\mathbf{P}^{*}(k)$. After processing all measurements $\mathbf{u}_{b}^{M}(k), b=\overline{1, B}$ a posteriori Gaussian p.d.f. $P\left(\mathbf{u}(k) \mid \mathbf{u}_{1}^{M}(k), \ldots, \mathbf{u}_{B}^{M}(k), \mathbf{U}^{M}(k-1)\right)$ and, accordingly, its parameters were determined.

Quasi-optimal algorithm (32)...(41) is nonlinear. Non-linear operations were performed when calculating a posteriori probabilities $P\left(a_{j_{b 0}}(k), a_{j_{b 1}}(k) \mid \mathbf{u}_{1}^{M}(k), \ldots, \mathbf{u}_{b}^{M}(k), \mathbf{U}^{M}(k-1)\right), j_{b 0}, j_{b 1}=\overline{1,2}$. A quasi-optimal device, that implements algorithm (32)...(41), contains four channels, which are related by general feedback.

\section{ALGORITHM EFFICIENCY ANALYSIS}

Analysis of the effectiveness of the developed quasi-optimal adaptive algorithm of estimating the UAV movement parameters (32)...(41) was carried out using statistical modeling.

The sensor network (Fig. 2) consists of eight sensors with coordinates (Table 1).

Table 1. Positions of wireless sensor networks sensors.

\begin{tabular}{ccccccccc}
\hline $\mathbf{S}$ & $\mathbf{1}$ & $\mathbf{2}$ & $\mathbf{3}$ & $\mathbf{4}$ & $\mathbf{5}$ & $\mathbf{6}$ & $\mathbf{7}$ & $\mathbf{8}$ \\
\hline $\mathrm{X}$ & 0 & 70.71 & 100 & 70.71 & 0 & -70.71 & -100 & -70.71 \\
\hline $\mathrm{Y}$ & 100 & 70.71 & 0 & -70.71 & -100 & -70.71 & 0 & 70.71 \\
\hline
\end{tabular}

For clarity of operation of the algorithm, a test trajectory of the UAV movement was formed (Fig. 2). The trajectory includes five sections. Abnormal measurement errors occur for:

- $S_{10}$ and $S_{11}$ in $k=20$ moment of time;

- $S_{20}$ and $S_{21}$ in $k=30$ moment of time;

- $S_{30}$ in $k=50$ moment of time;

- $S_{40}$ in $k=80$ moment of time; 
- $S_{31}$ in $k=60$ moment of time;

- $S_{41}$ in $k=90$ moment of time.

RMS error of measurement was $\sigma_{\varphi}=\sigma_{\theta}=0.8^{\circ}$, the rate of information receipt was $T=1 \mathrm{~s}$. Tests were carried out according to one hundred realizations $L=100$.

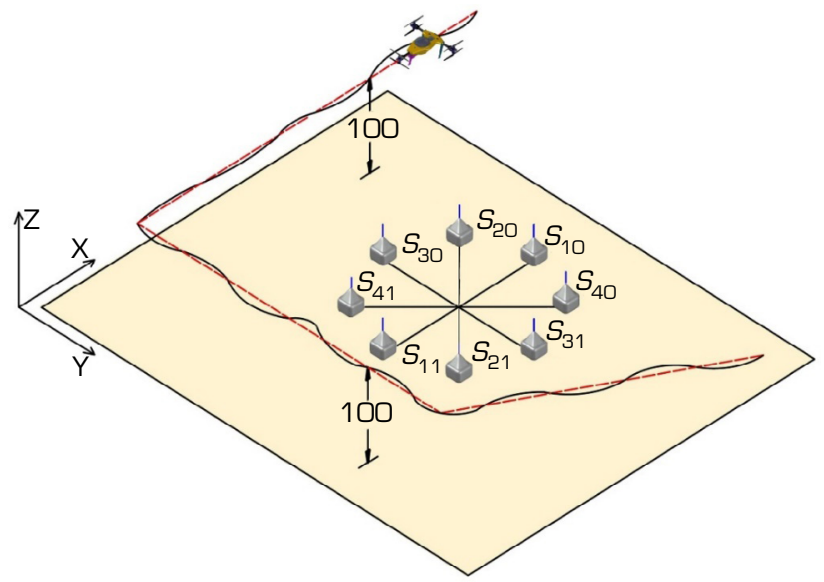

Figure 2. Configuration of sensor network of eight sensors and trajectory of unmanned aerial vehicles movement.

In the simulation, RMS of UAV acceleration change rate was assumed $\mathrm{a}=6 \mathrm{~m} / \mathrm{c}^{3}$ The probabilities of abnormal sensor measurements were the same $q_{2_{s}}=0.01, s=\overline{1, S}$, and the parameter $\gamma=7$.

Using the Monte Carlo method means $m_{\hat{\varepsilon}_{x}}(k), m_{\hat{\varepsilon}_{y}}(k), m_{\hat{\varepsilon}_{z}}(k)$ and RMS errors $\sigma_{\hat{\varepsilon}_{x}}(k), \sigma_{\hat{\varepsilon}_{y}}(k), \sigma_{\hat{\varepsilon}_{y}}(k)$ of estimation UAV locations were calculated by Eqs. 42-47.

$$
\begin{gathered}
m_{\hat{\varepsilon}_{x}}(k)=\frac{1}{L} \sum_{l=1}^{L} \hat{\varepsilon}_{x}^{l}(k)=\frac{1}{L} \sum_{l=1}^{L}\left(\hat{x}^{l}(k)-x(k)\right), k=\overline{1,130} \\
m_{\hat{\varepsilon}_{y}}(k)=\frac{1}{L} \sum_{l=1}^{L} \hat{\varepsilon}_{y}^{l}(k)=\frac{1}{L} \sum_{l=1}^{L}\left(\hat{y}^{l}(k)-y(k)\right), k=\overline{1,130} \\
m_{\hat{\varepsilon}_{z}}(k)=\frac{1}{L} \sum_{l=1}^{L} \hat{\varepsilon}_{z}^{l}(k)=\frac{1}{L} \sum_{l=1}^{L}\left(\hat{z}^{l}(k)-z(k)\right), k=\overline{1,130} \\
\sigma_{\hat{\varepsilon}_{x}}(k)=\sqrt{\frac{1}{L-1} \sum_{l=1}^{L}\left(\hat{\varepsilon}_{x}^{l}(k)-m_{\hat{\varepsilon}_{x}}(k)\right)^{2}}, k=\overline{1,130} \\
\sigma_{\hat{\varepsilon}_{y}}(k)=\sqrt{\frac{1}{L-1} \sum_{l=1}^{L}\left(\hat{\varepsilon}_{y}^{l}(k)-m_{\hat{\varepsilon}_{y}}(k)\right)^{2}}, k=\overline{1,130} \\
\sigma_{\hat{\varepsilon}_{z}}(k)=\sqrt{\frac{1}{L-1} \sum_{l=1}^{L}\left(\hat{\varepsilon}_{z}^{l}(k)-m_{\hat{\varepsilon}_{z}}(k)\right)^{2}}, k=\overline{1,130}
\end{gathered}
$$


Figure 3 shows RMS (curve 3) of coordinates $X, Y$ and $Z$ estimation errors calculated by the quasi-optimal algorithm using the correlation matrix $\hat{\mathbf{P}}_{b}(k)$. Also, Fig. 3 shows the actual (real) expected values (curve 1) $m_{\hat{\varepsilon}_{x}}(k), m_{\hat{\varepsilon}_{y}}(k), m_{\hat{\varepsilon}_{y}}(k)$ and the RMS (curve 2) $\sigma_{\hat{\varepsilon}_{x}}(k), \sigma_{\hat{\varepsilon}_{y}}(k), \sigma_{\hat{\varepsilon}_{z}}(k)$ of coordinate $X, Y$ and $Z$ estimation errors obtained experimentally by modeling. Calculated by a quasi-optimal algorithm and obtained experimentally RMS of estimation errors are close, which indicates the correct operation of the algorithm. In addition, Fig. 3 shows the actual RMS $\sigma_{\hat{\varepsilon}_{x}}(k), \sigma_{\hat{\varepsilon}_{y}}(k), \sigma_{\hat{\varepsilon}_{z}}(k)$ (curve 4) of estimation errors of coordinates $X, Y$ and $Z$, using the Kalman filter.
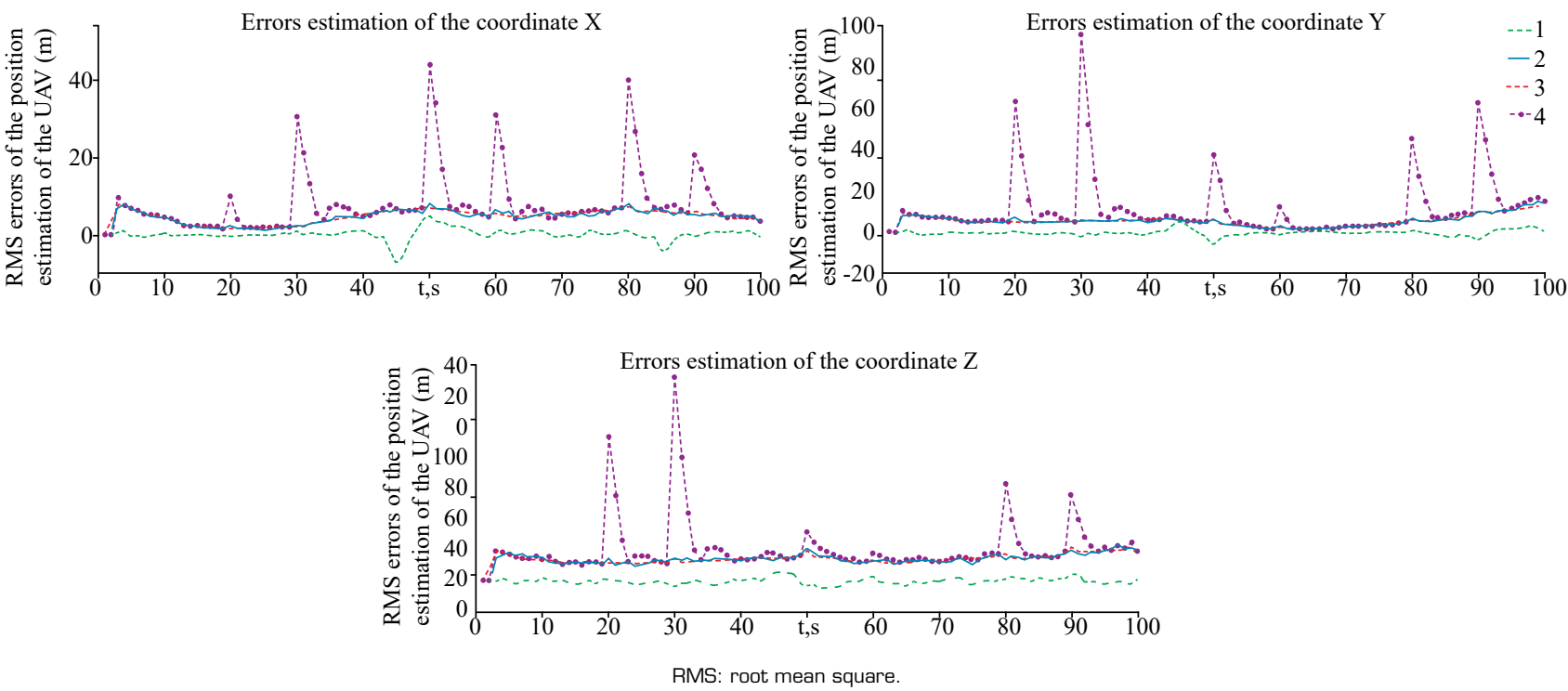

Figure 3. Characteristics of filtering error of unmanned aerial vehicles coordinates $X, Y$ and $Z$.

As follows from Fig. 3, the appearance of anomalous measurement errors led to uncontrolled increase of estimates errors of UAV movement parameters of Kalman filtering algorithm. The developed quasi-optimal algorithm for adaptive filtering of UAV movement parameters, based on AOA measurements of the sensor network, was resistant to the appearance of anomalous measurements.

\section{CONCLUSIONS}

An extended mixed process, including a continuous vector of UAV movement parameters and discrete switching variables that characterize the measurements type of sensors of the sensor network, has the Markov property, which makes it possible to synthesize a recurrent algorithm for calculating the posterior probability density of the mixed process. However, the implementation of an optimal real-time mixed process filtering algorithm encountered significant difficulties, associated with the need to integrate multidimensional p.d.f. and a large number of tested hypotheses, regarding the values of switching variables.

A quasi-optimal algorithm of adaptive filtering of UAV movement parameters, based on AOA measurements of the sensor network (32) ... (41), was obtained by linearizing the measurement Eqs. 2, 3 and 4. In this case, when the next measurement was received from each pair of sensors, a Gaussian approximation of a posteriori p.d.f. of the vector of the UAV movement parameters was performed. The quasi-optimal adaptive filter contained four channels which were related by general feedback. The implementation of the quasi-optimal algorithm did not require significant computational costs.

The quasi-optimal adaptive filtering algorithm (32)...(41) allows to eliminate the uncontrolled increase of estimates errors of the UAV movement parameters, caused by the appearance of anomalous measurements in comparison with the Kalman filter. 


\section{AUTHORS' CONTRIBUTION}

Conceptualization: Tovkach IO and Vasyliev VN; Methodology: Zhuk SY and Tovkach IO; Investigation: Neuimin OS and Tovkach IO; Writing - First Draft: Zhuk SY, Tovkach IO and Vasyliev VN.

\section{DATA AVAILABILITY STATEMENT}

All data sets were generated or analyzed in the current study.

\section{FUNDING}

Not applicable.

\section{ACKNOWLEDGMENTS}

Not applicable.

\section{REFERENCES}

Amiri R, Zamani H, Behnia F, Marvasti F (2016) Sparsity-aware target localization using TDOA/AOA measurements in distributed MIMO radars. ICT Express 2(1):23-27. https://doi.org/10.1016/j.icte.2016.02.002

Bar-Sholom Y., Xivo Rong L (1998) Estimation and tracking: principles, tech- techniques and software. Beaufort: YBS Publishing.

Chang CB, Athans M (1978) State estimation for discret systems with switching parameters. IEEE Transactions on Aerospace and Electronic Systems 14(3):418-425. https://doi.org/10.1109/TAES.1978.308603

Chen C-Y, Wu W-R (2018) Joint AoD, AoA, and channel estimation for MIMO-OFDM Systems. IEEE Transactions on Vehicular Technology 67(7):5806-5820. https://doi.org/10.1109/tvt.2018.2798360

Hou Y, Yang X, Abbasi Q (2018). Efficient AoA-based wireless indoor localization for hospital outpatients using mobile devices. Sensors 18(11):3698. https://doi.org/10.3390/s18113698

Kupriyanov AI, Sakharov AV (2007) Theoretical foundations of electronic warfare: Textbook. University Book.

Lacher A, Baron J, Rotner J, Balazs M (2019) Small unmanned aircraft: characterizing the threat. The MITRE Corporation. Available at: https://www.mitre.org/sites/default/files/publications/pr-18-3852-small-uas-characterizing-threat.pdf. Accessed on:

Sage E, Mels J (1971) Theory of estimation and its applications in communications and control. New York: McGraw Hill.

Sirota A, Kirsanov E (2006) The neural-network and statistical algorithms for estimating coordinates of a source of radio radiation in multi-position radio systems in the presence of abnormal errors of primary parameter measurement. Radioelectronics and Communications Systems 49(4):13-18. https://doi.org/10.3103/S0735272706040030 
Tang H, Park Y, Qiu T (2008) A TOA-AOA-based NLOS error mitigation method for location estimation. EURASIP Journal on Advances in Signal Processing, 682528. https://doi.org/10.1155/2008/682528

Tomic S, Beko M, Dinis R, Bernardo L 2018 On target localization using combined RSS and AoA measurements. Sensors 18(4):1266. https://doi.org/10.3390/s18041266

Tovkach IO, Neuimin OS, Zhuk SY (2018) Filtration of parameters of the UAV movement based on the RSS-measurement at the unknown power of the transmitter. In: 14th International Conference on Advanced Trends in Radioelecrtronics, Telecommunications and Computer Engineering (TCSET), 2018, p. 57-60. https://doi.org/10.1109/TCSET.2018.8336155

Tovkach IO, Zhuk SY (2019) Adaptive filtration of parameters of the UAV movement based on the TDOA-measurement sensor networks. Journal of Aerospace Technology and Management 11:e3519. https://doi.org/10.5028/jatm.v11.1062

Tovkach IO, Zhuk S (2020) Adaptive filtration of the UAV movement parameters based on the AOA-measurement sensor networks. International Journal of Aviation, Aeronautics, and Aerospace 7(3). https://doi.org/10.15394/ijaaa.2020.1497

Tovkach IO, Zhuk SY (2021) Filtration of UAV movement parameters based on the received signal strength measurement sensor networks in the presence of anomalous measurements of unknowln power at the transmitter. Journal of Aerospace Technology and Management 13:e0921. https://doi.org/10.1590/jatm.v13.1191

Tovkach IO, Zhuk SY, Reutska YY, Neuimin OS (2019) Estimation of radio source movement parameters based on TDOAand RSS- measurements of sensor network in presence of anomalous measurements. In: IEEE 39th International Conference on Electronics and Nanotechnology 778-783. https://doi.org/10.1109/ELNANO.2019.8783384

Tovkach IO, Zhuk SY, Neuimin OS, Chmelov VO (2020) Recurrent algorithm of passive location in sensor network by angle of arrival of a signal. IEEE 40th International Conference on Electronics and Nanotechnology 728-732. https://doi. org/10.1109/ELNANO50318.2020.9088740

U.S. Department of Homeland Security. Cybersecurity and Infrastructure Security Agency. Interagency Security Committee (2020) Protecting against the threat of unmanned aircraft systems (UAS). Interagency Security Committee. Available at: https://www.cisa.gov/sites/default/files/publications/Protecting\%20Against $\% 20$ the\%20Threat\%20of\%20Unmanned\%20 Aircraft\%20Systems\%20November\%202020_508c.pdf. Accessed on:

Watanabe F (2021) Wireless sensor network localization using AoA measurements with two-step error variance-weighted least squares. IEEE Access 9:10820-10828. https://doi.org/10.1109/ACCESS.2021.3050309

Yaacoub JP, Noura H, Salman O, Chehab A (2020) Security analysis of drones systems: attacks, limitations, and recommendations. Internet of Things 11:100218. https://doi.org/10.1016/j.iot.2020.100218

Zhang R, Liu J, Du X, Li B, Guizani M (2018) AOA-based three-dimensional multi-target localization in industrial WSNs for LOS conditions. Sensors 18(8):2727. https://doi.org/10.3390/s18082727

Zhuk SY (2020) Estimation of stochastic processes with random structure and markov switches in discreet time (review). Radioelectronics and Communications Systems. 63:505-520. https://doi.org/10.3103/S0735272720100015

Zhuk SY, Tovkach IO, Reutska YY (2019) Adaptive filtration of radio source movement parameters based on sensor network TDOA measurements in presence of anomalous measurements. Radioelectronics and Communications Systems. 62:61-71. https://doi.org/10.3103/S073527271902002X 